\title{
REVIEW
}

\section{Cancer heterogeneity is not compatible with one unique cancer cell metabolic map}

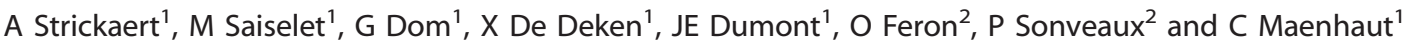

The Warburg effect and its accompanying metabolic features (anaplerosis, cataplerosis) are presented in textbooks and reviews as a hallmark (general characteristic): the metabolic map of cancer. On the other hand, research articles on specific tumors since a few years emphasize various biological features of different cancers, different cells in a cancer and the dynamic heterogeneity of these cells. We have analysed the research literature of the subject and show the generality of a dynamic, evolving biological and metabolic, spatial and temporal heterogeneity of individual cancers. We conclude that there is no one metabolic map of cancer but several and describe the two extremes of a panel from the hypoxic to the normoxic state. The implications for the significance of general 'omic' studies, and on therapeutic conclusions drawn from them and for the diagnostic use of fractional biopsies is discussed.

Oncogene (2017) 36, 2637-2642; doi:10.1038/onc.2016.411; published online 31 October 2016

\section{INTRODUCTION}

Cancer biology has led to the development of parallel concepts that provide a general framework; among them are: hallmarks of cancer, cancer metabolic map and cancer heterogeneity. There are few attempts to integrate these concepts. In this review, we outline them and their compatibility, and we show that there is no one unique metabolic map of a heterogeneous cancer. This is often acknowledged and discussed in specialized articles on cancer cell metabolism, but not in reviews and textbook chapters on cancer.

\section{HALLMARKS OF CANCER}

Hallmarks of cancer have been proposed as general concepts of the common properties of cancer. ${ }^{1}$ The first and the follow-up articles on the topic are most cited in the fundamental oncology literature. ${ }^{1,2}$ The concept of hallmarks has been used with different meanings in different derived articles. It could apply to cancer in general or to the idealized 'cancer cell' of the molecular biologists. The latter one often corresponds to our main research tool: cancer cell lines. However, cancer cell lines are poorly representative of cancer cells in vivo. ${ }^{3}$ Moreover, the reasoning on cell lines explains why one of the first steps of tumorigenesis, that is, dedifferentiation ${ }^{4}$ was not considered. The authors later pointed out that tumors are complex tissues, and emphasized the role of tumor microenvironment. ${ }^{2}$ Nevertheless, in many articles, the ambiguity about the object to which the concept is applied leads to misconceptions. ${ }^{4}$ Hallmarks are in fact properties of the cancer as a whole and of some of cancer cells sometimes.

Among the emerging hallmarks considered in 2011 was the reprogramming of energy metabolism. ${ }^{2,5,6}$ However, reviews and textbooks generally continue to present an idealized picture of the 'cancer cell' metabolism.'

\section{THE CANCER METABOLISM}

Since Warburg's original discovery, ${ }^{8}$ high levels of glycolysis and a low respiration rate, even in normoxic conditions, have been considered as a fundamental property and, sometimes, as a cause of cancer.

The 'Warburg effect' consists in an enhancement of glucose metabolism through glycolysis, often coupled to an inhibition of mitochondrial pyruvate oxidation, resulting in elevated lactate production in the presence of oxygen. For non-cancer cells, this is the normal pattern of metabolism in hypoxic conditions. ${ }^{9-12}$ In cancer cells, the Warburg effect can be coupled to an upregulation of cellular and mitochondrial glutamine uptake, which can, to some extent, compensate the reduced pyruvate availability at the mitochondrial level for adenosine triphosphate production and for biosynthesis (anaplerosis). It may also be coupled to an increased export to the cytosol of tricarboxylic acid cycle intermediates (cataplerosis) by which mitochondria feed pyruvate and citrate synthesis, to the activation of fatty acid metabolism, and to the synthesis of aminoacids and pentoses. ${ }^{12,13}$ This metabolic reprogramming is of course wider than the effect initially described by Warburg ${ }^{6}$ (Figure 1).

Metabolic reprogramming is mediated at the transcriptional and posttranscriptional level of metabolic enzymes and transporters. In particular, a significant part of the metabolic changes associated to the Warburg effect can be ascribed to hypoxiainducible transcription factors (HIF-1 and HIF-2). These factors are normally activated in hypoxia because of a reduction in the natural degradation rate of their a subunits that requires the presence of $\mathrm{O}_{2}$. HIFs are further controlled positively by oncogenes (for example, MYC) and negatively by tumor-

\footnotetext{
${ }^{1}$ Institut de Recherche Interdisciplinaire en Biologie Humaine et Moléculaire (IRIBHM), Université libre de Bruxelles (ULB), Brussels, Belgium and ${ }^{2}$ Pole of Pharmacology and Therapeutics, Institut de Recherche Expérimentale et Clinique (IREC), Université catholique de Louvain (UCL), Brussels, Belgium. Correspondence: Dr JE Dumont, Institut de Recherche Interdisciplinaire en Biologie Humaine et Moléculaire (IRIBHM), Université libre de Bruxelles (ULB), Campus Erasme, 808 Route de Lennik, Brussels 1070 Belgium. E-mail: jedumont@ulb.ac.be 


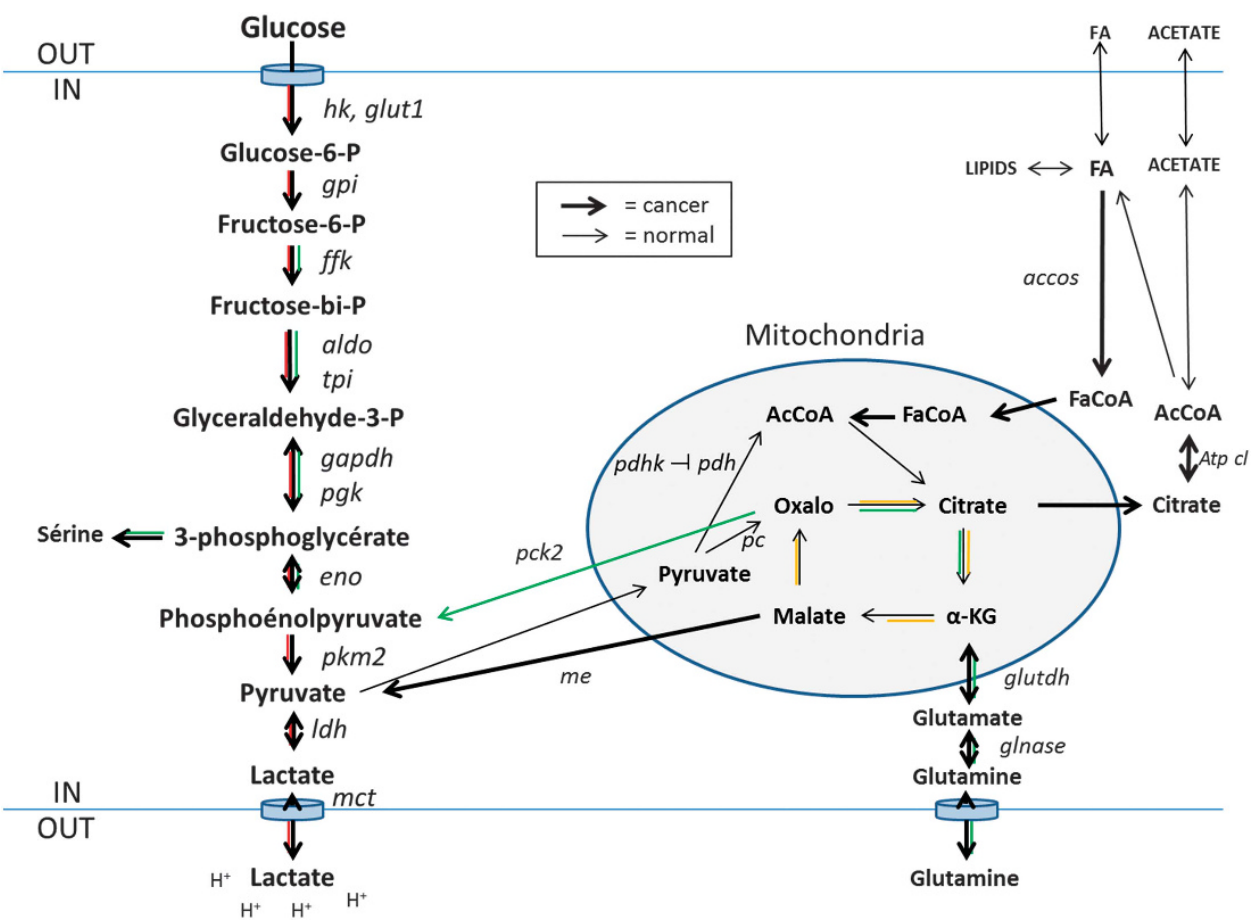

Figure 1. Simplified scheme of 'the metabolic map of cancer cell'. The classical normal cell metabolism in the presence of $\mathrm{O}_{2}$, from glucose to pyruvate, mitochondrial acetyl-CoA, and through the Krebs cycle to $\mathrm{CO}_{2}$ : black lines. The glycolytic Warburg pathway, from glucose to pyruvate and lactate: red lines. The pathways, from glutamine, acetate, and fatty acids to aminoacids, nucleotides and fat: green lines. The Krebs cycle: yellow lines. accos, acetylcoA synthase; aldo, aldolase; Atp cl, adenosine triphosphate citrate lyase; eno, enolase; FaCoA, fatty acyl$\mathrm{CoA}$; ffk, phosphofructokinase; gapdh, glyceraldehyde phosphate dehydrogenase; glnase, glutaminase; glut1, glucokinase; glutdh, glutamic acid dehydrogenase; gpi, glucose phosphate isomerase; hk, hexokinase; ldh, lactate dehydrogenase; mct, monocarboxylic acid transporter; me, malic enzyme; oxalo, oxaloacetate; pc, pyruvate carboxylase; pdh, pyruvate dehydrogenase; pdhk, pyruvate dehydrogenase kinase; pck2, pyruvate carboxykinase; pgk, phosphoglycerate kinase; pkm2, pyruvate kinase $\mathrm{m} 2$; tpi, triose phosphate isomerase.

suppressor genes. $5,6,10,14$ Adaptation to hypoxia, and notably HIF activation, further promotes invasion and metastasis, immune resistance by recruitment of Treg lymphocytes and tumor macrophages and so on. ${ }^{10,15,16}$

This common phenotype of cancer cells metabolic transformation is considered to be essential for their growth and progression. ${ }^{5}$ In cancer cell lines, it also promotes resistance to anoikis. ${ }^{17}$ When predominant, the glycolytic activity demonstrated by high-lactate levels in tumors predicts the likelihood of tumor aggressiveness and metastasis in several cancer types. ${ }^{18-22}$

\section{DYNAMIC HETEROGENEITY OF CANCER}

Although tumor heterogeneity has become a byword, our language, reasoning and investigations often assume homogeneous systems. As a result, a misleading tendency for molecular biologists, accustomed to work on homogenous populations of cancer cell lines, is to extrapolate their observations to in vivo tumors. ${ }^{3}$ A same simplification entails the interpretation by many researchers of the concept of hallmarks of cancers and map of cancer metabolism, with an erroneous assumption of homogeneity. Common tools of cancer research, such as measurements on biopsies or 'omic studies' on pieces of tumors (DNA sequencing, genes and protein expressions, metabolomics and so on), further imply the same assumptions. In fact, pathologists familiar with tissue sections have always known about tumor heterogeneity.

The concept of tumor heterogeneity encompasses transient and quasi-permanent situations. There are at least the following four aspects in this heterogeneity (Figure 2):
(1) Cell populations (stromal cells, different tumor clones and so on).

(2) Cell localization (for example, close or distant from other cells, from perfused blood vessels, from $\mathrm{O}_{2}$ and nutrient resources and so on).

(3) Timing in signal transduction from minutes for direct and posttranslational controls, to hours for transcriptional controls, to days and months for epigenetic mechanisms, and permanence for genetic effects.

(4) Patterns of metabolism and cell biology (for example, epithelial mesenchymal transition; vide infra).

For cell population heterogeneity, the coexistence of invading tumor cells and normal cells, the presence in the tumor mass of stromal cells such as cancer-associated fibroblasts, of macrophages, lymphocytes and endothelial cells are prime examples. This cellular heterogeneity varies much from one type of cancer to another. For instance, thyroid papillary and anaplastic carcinomas may contain as many associated cells as cancer cells (CAFs as cancer-associated fibroblasts in PTC, and TAM as tumor-associated macrophages in ATC), while follicular carcinomas are more homogeneous and are populated mostly by cancer cells. In papillary thyroid carcinomas, the proportion of cancer-associated cells correlates with malignity. ${ }^{23,24}$

In the cancer cells population, clonal evolution gives rise to clones that progressively accumulate genetic mutations ${ }^{25,26}$ and epigenetic alterations. Heterogeneity of different clones may favor cooperation for growth ${ }^{27}$ and metastasis. ${ }^{28}$ Also, even among the cancer cells themselves, only a minority may be responsible for cancer progression. In breast cancers, a comparison of cells and 
a

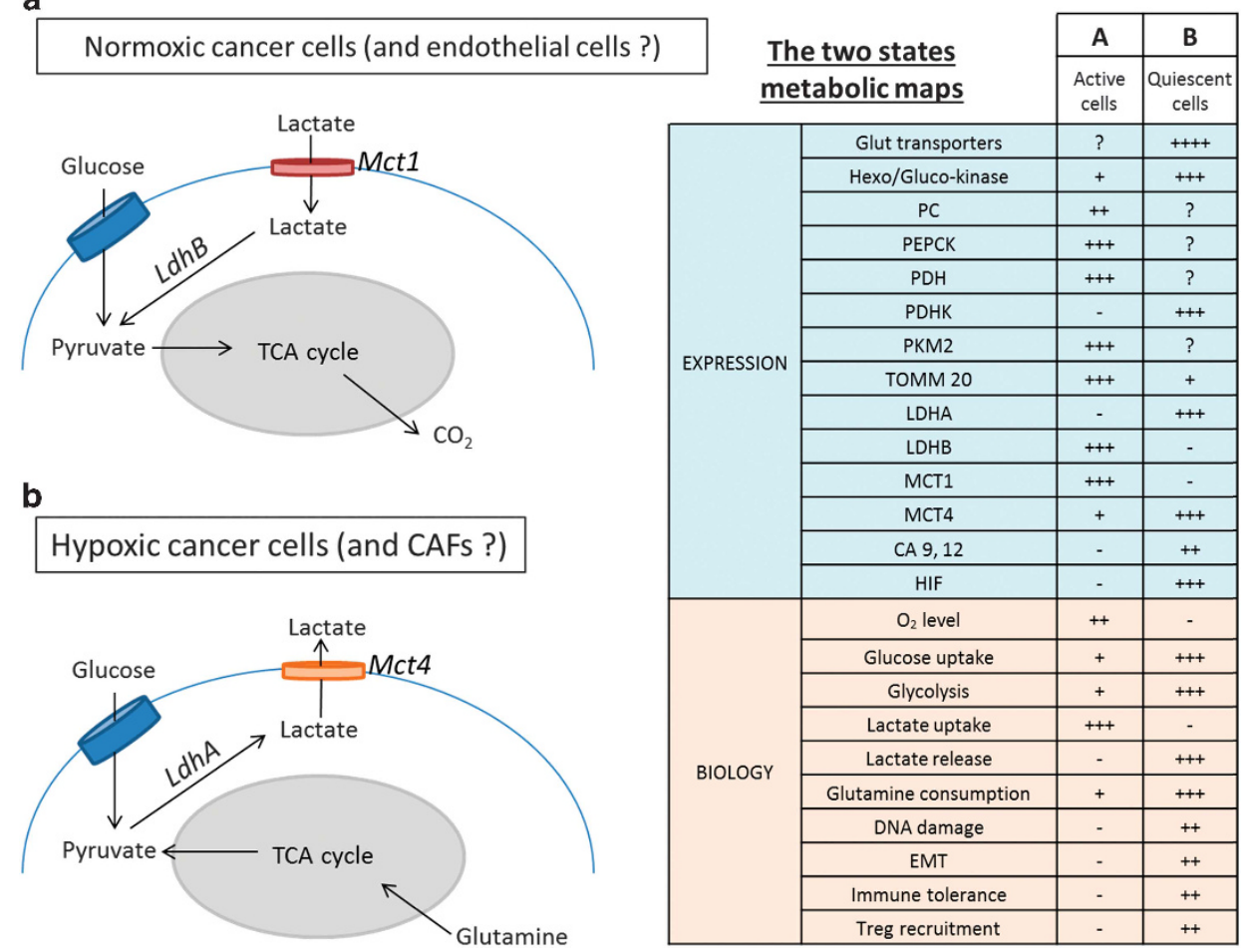

Figure 2. Diversity of cells in cancer. EMT, epithelial mesenchymal transition.

tumor duplication rates has shown that a majority of the cancer cells die, whereas only a minority of them drives cancer growth. ${ }^{29,30}$ This may, but does not necessarily, reflect a Darwinian cell evolution. ${ }^{31}$ Similarly, in normal tissues, cells may display somatic mosaicism. ${ }^{32}$ Finally, even in a homogenous cell line in culture, stochastic state transitions give rise to phenotypic equilibrium in populations. ${ }^{33}$

Concerning the different cell localizations, they depend on an evolving vascular development, perfusion abnormalities s $^{34,35}$ and on tumor growth. Similar cells from normally oxygenated (normoxic) areas will, when distant from functional blood vessels capable of carrying red blood cells, progressively degenerate into slightly to deeply hypoxic areas. Shifts in cell behavior and protein patterns imposed by the environment, for example by lactate or $\mathrm{H}^{+}$as signals, ${ }^{36}$ may promote mechanisms of cell migration, amoeboid or collective, ${ }^{37,38}$ angiogenesis and immunosuppression. ${ }^{36,39}$ They may result from epigenetic, transcriptional, translational or posttranslational mechanisms, and be more or less reversible. ${ }^{38,40,41}$ Thus, tumors have been described as evolving ecosystems. ${ }^{42}$ Changes imposed by the microrenvironment may also exist permanently, even in genetically homogenous populations of unicellular organisms. ${ }^{43}$ Permanent and transient switches are often objectivated by single cell RNA sequencing. ${ }^{44}$ This intratumoral heterogeneity evolves through space and time. ${ }^{45}$

Finally, the time issue cannot be dissociated from the concept of cycles. Cancer cells may for instance be in an hypoxic environment for only a transient period of time either because angiogenesis is developing in response to low $\mathrm{pO}_{2}$ and reoxygenation will progressively occur, or because the highly immature tumor vasculature that was not functional is suddenly re-perfused because of an hemodynamic change (for example, red blood cells that were plugged in a poorly organized tumor capillary are suddenly re-mobilized). ${ }^{46}$ These phenomena have been proposed to repeat themselves with different frequencies. ${ }^{35}$ Importantly, these cycles of hypoxia/reoxygenation (more exactly, variations between deep and moderate hypoxia) have been shown to influence the phenotype of cancer cells but also endothelial cells. ${ }^{4748}$ The gene program induced by intermittent or cycling hypoxia is very different from that corresponding to continuous hypoxia. Of note, a transcriptomic signature derived from cycling hypoxia was recently documented to have a very efficient prognostic potential to discriminate between low- and high-risk breast cancer patients, ${ }^{49}$ indicating that the capacity of tumor to adapt to heterogeneous conditions of oxygenation and perfusion could be linked to cancer aggressiveness.

\section{CONSEQUENCES OF HETEROGENEITY}

Ignoring tumor heterogeneity has at least two consequences: (a) it may lead to erroneous interpretations of research and clinical data, and (b) it masks the complex network of cellular interactions that govern tumor behavior.

In the first case, increase in the global measurement of the content of one RNA or enzyme in some cells may mask the decrease of the same RNA or enzyme in other cells and conversely. Thus, one may find in global data of a set of cells evidence of activation of a reaction in opposite senses (lactate influx and efflux, coincrease of MCT4 and MCT8, of LDH A and LDH $B$ and so on).$^{50}$ In one cell, this would constitute a futile cycle, which in a competitive environment could be suicidal. However, a coexpression of opposite enzymes whose activity is controlled posttrancriptionnally would provide the possibility of rapid switches. The metabolic profile of a cell has to make biological sense. As a typical example of possible misinterpretations, the heterogeneity of a renal carcinoma led to both good and bad prognosis signatures by sequencing different fragments of the same piece of tissue. ${ }^{51}$

On the other hand, the coexistence of different types of cells and of similar cells with different metabolic phenotypes in response to different environments may be essential for tumor progression. ${ }^{52}$ For example, there is a strong interrelation of cancer cells and TAMs in thyroid anaplastic carcinoma cells, ${ }^{53}$ and CAFs in thyroid papillary carcinoma, ${ }^{23,24}$ and of fibroblasts with 
breast and ovarian cancer cells and so on. In the latter cases, this association in desmoplasia has been shown to be a crucial element in cancer progression. ${ }^{54}$ Breast cancer heterogeneity is a strong negative predictor of outcome, which suggested that Darwinian competition would be beneficial for the tumor. ${ }^{55}$

\section{HETEROGENEITY AND METABOLISM}

Among cancer cells themselves, clonal evolution may lead to diversity and heterogeneity, and variations in location and time may further cause transient or permanent differences. Cancer cells are notoriously resourceful, ${ }^{56}$ and may use this diversity. For instance, they can switch mitochondrial substrates from glucose to glutamine and conversely through transcriptional control. ${ }^{13,57}$

Adaptability is especially relevant to cancer cell metabolism, with short-term and permanent adaptations. At the molecular level, the Warburg effect encompasses increased glucose metabolism to lactate (glycolysis) and to the pentose phosphate pathway. Anaplerosis and cataplerosis are not strictly linked to the Warburg effect. These metabolic changes involve: (a) activation and/or upregulation of glucose transporters, hexokinases and glycolytic enzymes (for example, PKM2), lactate dehydrogenases (LDH) and transporters of lactate (for example, MCT) and protons (for example, carbonic anhydrase IX); and (b) downregulation or inactivation by PDK2 of pyruvate dehydrogenase and so on. Fuel availability may itself determine the metabolic preference: cancer cells that produce lactate as long as glucose is available may recapture lactate when glucose is lacking, lactate being then reconverted in pyruvate to fuel the tricarboxylic acid cycle. ${ }^{58}$

Adaptation to $\mathrm{O}_{2}$ availability can be rapid (minutes), involving direct and posttranslational regulations, or slow with chronic or delayed changes involving transcriptional, epigenetic and genetic mechanisms. Both types of responses can be evaluated with labeled glucose in culture and in vivo, ${ }^{59}$ giving an integrated global picture. However, even for clonal cells, the relation between hypoxic and normoxic areas of a cancer depends on variations in blood flow, which may be rapid. ${ }^{34,35,60}$ This can be tested in vivo, and, indeed, an increased $\mathrm{O}_{2}$ supply rapidly decreases glucose uptake in tumors. ${ }^{61}$ The delayed or chronic type of cell adaptation to hypoxia is often demonstrated a posteriori by histology and molecular biology on tumors, ${ }^{5,10,14,19,62}$ or on metastases. ${ }^{63}$

Chronic regulations of metabolic heterogeneity can be studied directly in vitro in culture, and their consequences can be evaluated in vivo, notably on biopsies. In general, the $\mathrm{O}_{2}$ and glucose supplies of a given tumor are co-modulated by the blood supply of the tumor, to the exception of blood vessels that are perfused by plasma only but do not carry red blood cells.
The transport capacity of blood vessels is clearly reduced by the chaotic state of the tumor microvasculature. ${ }^{35,60}$ However, the cellular consequences of a reduced blood supply are very different when considering $\mathrm{O}_{2}$ and glucose availability. Arterial $5 \% \mathrm{O}_{2}$ corresponds to $10 \mu \mathrm{mol} / \mathrm{l}$, whereas $900 \mathrm{mg} / \mathrm{l}$ of glucose corresponds to $5000 \mu \mathrm{mol} / \mathrm{l}$. Oxygen is poorly soluble in water, hence in the plasma, whereas glucose is. Thus, oxygen is a more limiting resource when compared with glucose. Consequently, cells distant from the well-perfused capillaries will be hypoxic but could still be supplied in glucose. The already low $\mathrm{pO}_{2}$ near capillaries decreases with distance and thus often with the size of the tumor, ${ }^{64}$ with sometimes more than $60 \%$ of the cells being hypoxic in established tumors. ${ }^{65}$ The effects of low $\mathrm{O}_{2}$ and the consequent limitation of oxidative phosphorylation prevent the growth of breast cancer cells, but less so of cancer-associated fibroblasts. ${ }^{66}$

The coexistence in the same tumor of hypoxic and normoxic cells has been modeled in vitro. It leads to a symbiosis between the two populations of largely specialized cells. ${ }^{52}$ In 3D structures in vitro, and in experimental tumors in vivo, the normoxic cells are at the periphery and the hypoxic cells are in the center of a spheroid. This suggests a metabolic map for each type of cell. ${ }^{67}$ In these maps, hypoxic cells produce lactate from glucose (anaerobic glycolysis) and normoxic cells take it up and oxidize it (Figure 3). Similar metabolic relationships can also exist between cancer and stromal cells. Metabolic commensalism has been described with cancer cells releasing reactive oxygen species that promote a glycolytic metabolic pathway in CAFs, that in turn provides the oxidative cancer cells with lactate and ketone bodies. ${ }^{20,68,69}$ CAFs have also been shown to be major contributors to extracellular acidification, promoting the epithelial mesenchymal transition of the tumor cells. ${ }^{70}$

There would therefore exist at least two different archetype metabolic maps for a given cancer, and there might also be intermediates. $^{28,36}$ The metabolic maps indicate global enzymatic activities, which reflect effects on their activity and on the expression of different isoforms. The former are rapid and will only be demonstrated grossly with labeled substrates. ${ }^{59}$ The latter results from controls at translational, transcriptional, epigenetic and even genetic levels. ${ }^{10}$

The different components of cancer metabolism (glucose, glutamine, lipid and lactate metabolism, pyruvate carboxylation, anaplerosis/cataplerosis and so on) may not always be tightly linked, for example, the mitochondrial use of glutamine may occur independently of $\mathrm{O}_{2}$ consumption. ${ }^{57,71,72}$ However, crosstalks may exist. For example, intracellular lactate promotes glutamine uptake and metabolism in oxidative cancer cells ${ }^{73}$ and conversely, anaplerosis, the conversion of pyruvate to oxaloacetate by

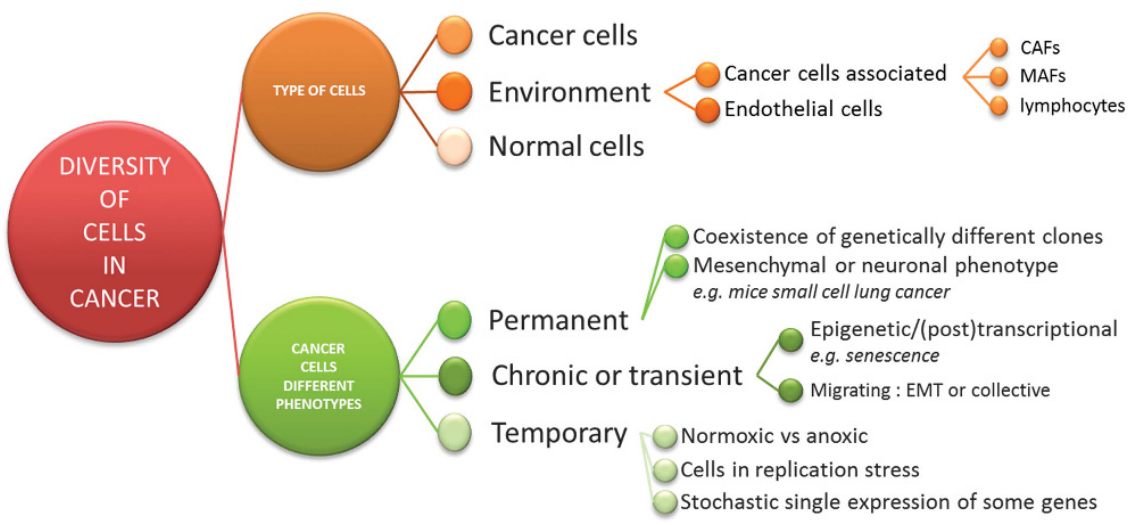

Figure 3. The two states metabolic map. Expression and Biology CA, carbonic anhydrase; CAF, cancer-associated fibroblasts; LDH, lactate dehydrogenase; HIF, hypoxia inducible factor; Lox, lysyl oxidase; MCT, monocarboxylic transporter; PDH, pyruvate dehydrogenase; PDHK, pyruvate dehydrogenase kinase; PKM2, pyruvate kinase M2; TOMM20, translocase of outer mitochondrial membrane 20. 
pyruvate carboxylase, allows glucose to fuel glutamate synthesis. ${ }^{74}$ Potential contributions of non-glucose nutrients in well-perfused tumor areas should also be considered ${ }^{59}$ together with the influence of acidosis that was recently shown to shift tumor metabolism from glucose to glutamine and fatty acid metabolism. ${ }^{75}$ In fact the great metabolic flexibility of cancer cells in vivo and in vitro multiplies the number of possible coexisting metabolic maps. ${ }^{76}$

The possibility of intermediate states between metabolic phenotypes is doubtful as they would represent bidirectional pathways, that is, wasteful futile cycles. It is therefore probable that all or none controls (for example, by positive feedbacks) would impose switches rather than gradual metabolic states. Even if it is not mentioned, published histochemical studies indeed rather suggest clean demarcations between tumor aerobic and anaerobic areas rather than progressive transitions. ${ }^{10,63}$

The validity of the concepts of hypoxic and normoxic areas in in vivo tumors is supported by differential uptakes of glucose in hypoxic and vascularized regions of the tumors, ${ }^{59,77}$ by the use of markers of hypoxia such as pimonidazole and carbonic anhydrase IX staining as well as ${ }^{18}$ Fluoromisonidazole $\left({ }^{18} \mathrm{~F}-\mathrm{MISO}\right)$, and by direct $\mathrm{pO}_{2}$ measurements and electron paramagnetic resonance oxymetry. ${ }^{36}$ Dynamic contrast-enhanced MRI combined with ${ }^{13} \mathrm{C}$ glucose infusion suggests that the relatively stable perfusion state of lung tumors is a major contributor of metabolic variability. ${ }^{59}$ In fact, there is a correlation between this duality and tumor aggressiveness in urothelial bladder cancers. ${ }^{78}$ Of course, the situation may vary from one tumor type to another.

\section{CONCLUSIONS}

The dynamic heterogeneity of metabolic phenotypes in tumors and metastases has consequences. On the conceptual point of view, the unique cancer metabolic map gives an erroneous oversimplified static description of the heterogeneous, versatile, adaptable cancer metabolism. From the diagnostic standpoint, the metabolic pattern of a cancer biopsy cannot be considered as representative of the whole tumor. With regard to therapeutics, heterogeneity raises problems. ${ }^{45}$ Transient and locationdependent phenotypes may not be considered as suitable targets, unless largely predominant or attacked jointly. ${ }^{36}$ More interesting features may be the chronic ones, such as proliferation targeted by recurrent chemo and radiotherapy.

\section{CONFLICT OF INTEREST}

The authors declare no conflict of interest.

\section{ACKNOWLEDGEMENTS}

The work of PS and OF is supported by EU Horizon2020 Marie Skłodowska-Curie Innovative Training Networks (ITN No. 642623 RADIATE), Interuniversity Attraction Pole (IAP) grant \#UP7-03 from the Belgian Science Policy Office (Belspo), an Action de Recherche Concertée from the Communauté Française de Belgique (ARC 14/19-058), the Belgian Fonds National de la Recherche Scientifique (F.R.S.-FNRS), the Télévie, and the Belgian Fondation contre le Cancer (2012-186). Pierre Sonveaux is a Research Associate of the F.R.S.-FNRS. Other authors of the University of Brussels are supported by the Belgian Fonds National de la Recherche Scientifique, Plan Cancer (action 29) and Welbio.

\section{REFERENCES}

1 Hanahan D, Weinberg RA. The hallmarks of cancer. Cell 2000; 100: 57-70.

2 Hanahan D, Weinberg RA. Hallmarks of cancer: the next generation. Cell 2011; 144: 646-674.

3 van Staveren WCG, Solis DY, Hebrant A, Detours V, Dumont JE, Maenhaut C Human cancer cell lines: experimental models for cancer cells in situ? For cancer stem cells? Biochim Biophys Acta 2009; 1795: 92-103.
4 Floor SL, Dumont JE, Maenhaut C, Raspe E. Hallmarks of cancer: Of all cancer cells, all the time? Trends Mol Med 2012; 18: 509-515.

5 Cairns R, Harris I, Mak T. Regulation of cancer cell metabolism. Nat Rev Cancer 2011; 11: 85-95.

6 Ward PS, Thompson CB. Metabolic reprogramming: a cancer hallmark even warburg did not anticipate. Cancer Cell 2012; 21: 297-308.

7 Ferreira LMR, Hebrant A, Dumont JE. Metabolic reprogramming of the tumor. Oncogene 2012; 31: 3999-4011.

8 Warburg O, Wind F, Negelein El. Killing-off of tumor cells in vitro. J Gen Physiol 1927; 8: 519-530.

9 Feron O. Pyruvate into lactate and back: from the Warburg effect to symbiotic energy fuel exchange in cancer cells. Radiother Oncol 2009; 92: 329-333.

10 Porporato PE, Dhup S, Dadhich RK, Copetti T, Sonveaux P. Anticancer targets in the glycolytic metabolism of tumors: a comprehensive review. Front Pharmacol 2011; 2: 49.

11 Payen VL, Brisson L, Dewhirst MW, Sonveaux P. Common responses of tumors and wounds to hypoxia. Cancer J 2015; 21: 75-87.

12 Currie E, Schulze A, Zechner R, Walther TC, Farese RV. Cellular fatty acid metabolism and cancer. Cell Metab 2013; 18: 153-161.

13 Corbet C, Feron O. Metabolic and mind shifts: from glucose to glutamine and acetate addictions in cancer. Curr Opin Clin Nutr Metab Care 2015; 18: 346-353.

14 Semenza GL. HIF-1 mediates metabolic responses to intratumoral hypoxia and oncogenic mutations. J Clin Invest 2013; 123: 3664-3671.

15 Rivera L, Bergers G. Location, location, location: macrophage positioning within tumors determines pro- or antitumor activity. Cancer Cell 2013; 24: 687-689.

16 Rankin EB, Giaccia AJ. Hypoxic control of metastasis. Science 2016; 352: 175-180.

17 Kamarajugadda S, Stemboroski L, Cai Q, Simpson NE, Nayak S, Tan M et al. Glucose oxidation modulates anoikis and tumor metastasis. Mol Cell Biol 2012; 32: 1893-1907.

18 Walenta S, Wetterling M, Lehrke M, Schwickert G, Sundfør K, Rofstad EK et al. High lactate levels predict likelihood of metastases tumor recurrence, and restricted patient survival in human cervical cancers. Cancer Res 2000; 60: 916-921.

19 Walenta S, Mueller-Klieser WF. Lactate: mirror and motor of tumor malignancy. Semin Radiat Oncol 2004; 14: 267-274.

20 Bonuccelli G, Tsirigos A, Whitaker-Menezes D, Pavlides S, Pestell RG, Chiavarina B et al. Ketones and lactate 'fuel' tumor growth and metastasis: evidence that epithelial cancer cells use oxidative mitochondrial metabolism. Cell Cycle 2010; 9: 3506-3514.

21 Lee GH, Kim DS, Chung MJ, Chae SW, Kim HR, Chae HJ. Lysyl oxidase-like-1 enhances lung metastasis when lactate accumulation and monocarboxylate transporter expression are involved. Oncol Lett 2011; 2: 831-838.

22 Draoui N, Feron O. Lactate shuttles at a glance: from physiological paradigms to anti-cancer treatments. Dis Model Mech 2011; 4: 727-732.

23 Jolly LA, Novitskiy S, Owens P, Massoll N, Cheng N, Fang W et al. Fibroblastmediated collagen remodeling within the tumor microenvironment facilitates progression of thyroid cancers driven by brafv600e and pten loss. Cancer Res 2016; 76: 1804-1813.

24 Tarabichi M, Antoniou A, Le Pennec S, Gacquer D, de Saint Aubain N, Craciun L et al. Higher proliferation of stroma and tumor cells generates complex $3 \mathrm{D}$ microvolumes in BRAFV600E-mutant papillary thyroid carcinomas. J Pathol 2016 submitted.

25 Andor N, Graham TA, Jansen M, Xia LC, Aktipis CA, Petritsch C et al. Pan-cancer analysis of the extent and consequences of intratumor heterogeneity. Nat Med 2015; 22: 105-113.

26 Zhang Xiaoyan, Marjani Sadie L, Hu Zhaoyang, Weissman Sherman M, Xinghua Pan SW. Single-cell sequencing for precise cancer research: progress and prospects. Cancer Res. 2016; 76: 1305-1312.

27 Cleary AS. Subclone cooperation maintains tumor growth. Science (80-) 2015; 350: 1174-1175.

28 Calbo J, van Montfort E, Proost N, van Drunen E, Beverloo HB, Meuwissen R et al. A functional role for tumor cell heterogeneity in a mouse model of small cell lung cancer. Cancer Cell 2011; 19: 244-256.

29 Bhatia S, Frangioni JV, Hoffman RM, lafrate AJ, Polyak K. The challenges posed by cancer heterogeneity. Nat Biotechnol 2012; 30: 604-610.

30 Malaise EP, Chavaudra N, Tubiana M. The relationship between growth rate, labelling index and histological type of human solid tumours. Eur J Cancer 1973; 9: 305-312.

31 Ling S, Hu Z, Yang Z, Yang F, Li Y, Chen Q et al. Correction for Ling et al. Extremely high genetic diversity in a single tumor points to prevalence of non-Darwinian cell evolution. Proc Natl Acad Sci USA 2016; 113: E663-E663.

32 Fernández LC, Torres M, Real FX. Somatic mosaicism: on the road to cancer. Nat Rev Cancer 2016; 16: 43-55.

33 Gupta PB, Fillmore CM, Jiang G, Shapira SD, Tao K, Kuperwasser C et al. Stochastic state transitions give rise to phenotypic equilibrium in populations of cancer cells. Cell 2011; 146: 633-644. 
34 Cardenas-Navia LI, Mace D, Richardson RA, Wilson DF, Shan S, Dewhirst MW. The pervasive presence of fluctuating oxygenation in tumors. Cancer Res 2008; 68: 5812-5819.

35 Dewhirst MW. Relationships between cycling hypoxia, HIF-1, angiogenesis and oxidative stress. Radiat Res 2009; 172: 653-665.

36 Marchiq I, Pouysségur J. Hypoxia, cancer metabolism and the therapeutic benefit of targeting lactate/H+ symporters. J Mol Med 2016; 94: 155-171.

37 Friedl P. Immunological techniques: dynamic imaging of the immune system. Curr Opin Immunol 2004; 16: 389-393.

38 Friedl P, Alexander S. Cancer invasion and the microenvironment: plasticity and reciprocity. Cell 2011; 147: 992-1009.

39 Hirschhaeuser F, Sattler UGA, Mueller-Klieser W. Lactate: a metabolic key player in cancer. Cancer Res 2011; 71: 6921-6925.

40 Dalerba P, Kalisky T, Sahoo D, Rajendran PS, Rothenberg ME, Leyrat AA et al. Single-cell dissection of transcriptional heterogeneity in human colon tumors. Nat Biotechnol 2011; 29: 1120-1127.

41 Avery SV. Microbial cell individuality and the underlying sources of heterogeneity. Nat Rev Microbiol 2006; 4: 577-587.

42 Tabassum DP, Polyak K. Tumorigenesis: it takes a village. Nat Rev Cancer 2015; 15: 473-483.

43 Snijder B, Pelkmans L. Origins of regulated cell-to-cell variability. Nat Rev Mol Cell Biol 2011; 12: 119-125.

44 Tirosh I, Izar B, Prakadan SM, Wadsworth MH, Treacy D, Trombetta JJ et al. Dissecting the multicellular ecosystem of metastatic melanoma by single-cell RNA-seq. Science (80-) 2016; 352: 189-196.

45 Swanton C. Intratumour heterogeneity: evolution through space and time an evolutionary perspective on cancer heterogeneity. Cancer Res 2013; 72: 4875-4882.

46 Polet F, Feron O. Endothelial cell metabolism and tumour angiogenesis: glucose and glutamine as essential fuels and lactate as the driving force. $J$ Intern Med 2013; 273: 156-165.

47 Daneau G, Boidot R, Martinive P, Feron O. Identification of cyclooxygenase-2 as a major actor of the transcriptomic adaptation of endothelial and tumor cells to cyclic hypoxia: effect on angiogenesis and metastases. Clin Cancer Res 2010; 16: 410-419.

48 Martinive P, Defresne F, Quaghebeur E, Daneau G, Crokart N, Grégoire V et al. Impact of cyclic hypoxia on HIF-1a regulation in endothelial cells - new insights for anti-tumor treatments. FEBS J 2009; 276: 509-518.

49 Boidot R, Branders S, Helleputte T, Rubio LI, Dupont P, Feron O. A generic cycling hypoxia-derived prognostic gene signature: application to breast cancer profiling. Oncotarget 2014; 5: 6947-6963.

50 Macaulay IC, Voet T. Single cell genomics: advances and future perspectives. PLoS Genet 2014; 10: e1004126.

51 Gerlinger M, Rowan AJ, Sc B, Horswell S, Math M, Larkin J et al. Intratumor heterogeneity and branched evolution revealed by multiregion sequencing. N Engl J Med 2016; 366: 883-892.

52 Nakajima EC, Van Houten B. Metabolic symbiosis in cancer: refocusing the Warburg lens. Mol Carcinog 2013; 52: 329-337.

53 Caillou B, Talbot M, Weyemi U, Pioche-Durieu C, Ghuzlan A, Bidart JM et al. Tumorassociated macrophages (TAMs) form an interconnected cellular supportive network in anaplastic thyroid carcinoma. PLoS One 2011; 6: e22567.

54 Yeung T-L, Leung CS, Yip K-P, Au Yeung CL, Wong STC, Mok SC. Cellular and molecular processes in ovarian cancer metastasis. A review in the theme: cell and molecular processes in cancer metastasis. Am J Physiol Cell Physiol 2015; 309: C444-C456.

55 Natrajan R, Sailem H, Mardakheh FK, Arias Garcia M, Tape CJ, Dowsett M et al. Microenvironmental heterogeneity parallels breast cancer progression: a histology-genomic integration analysis. PLoS Med 2016; 13: 1-19.

56 Tarabichi M, Antoniou A, Saiselet M, Pita JM, Andry G, Dumont JE et al. Systems biology of cancer: entropy, disorder, and selection-driven evolution to independence, invasion and "swarm intelligence". Cancer Metastasis Rev 2013; 32: 403-421.

57 Daye D, Wellen KE. Metabolic reprogramming in cancer: unraveling the role of glutamine in tumorigenesis. Semin Cell Dev Biol 2012; 23: 362-369.

58 Boidot R, Veǵran F, Meulle A, Le Breton A, Dessy C, Sonveaux P et al. Regulation of monocarboxylate transporter MCT1 expression by p53 mediates inward and outward lactate fluxes in tumors. Cancer Res 2012; 72: 939-948.
59 Hensley CT, Faubert B, Yuan Q, Lev-Cohain N, Jin E, Kim J et al. Metabolic heterogeneity in human lung tumors. Cell 2016; 164: 681-694.

60 Ostergaard L, Tietze A, Nielsen T, Drasbek KR, Mouridsen K, Jespersen SN et al. The relationship between tumor blood flow, angiogenesis, tumor hypoxia, and aerobic glycolysis. Cancer Res 2013; 73: 5618-5624.

61 Neveu MA, Bol V, Bol A, Bouzin C, Grégoire V, Feron O et al. The increase in tumor oxygenation under carbogen breathing induces a decrease in the uptake of [18F]fluoro-deoxy-glucose. Radiother Oncol 2015; 116: 400-403.

62 Doherty J, Cleveland J. Targeting lactate metabolism for cancer therapeutics. J Clin Invest 2013; 123: 3685-3692.

63 Turtoi A, Blomme A, Debois D, Somja J, Delvaux D, Patsos G et al. Organized proteomic heterogeneity in colorectal cancer liver metastases and implications for therapies. Hepatology 2014; 59: 924-934.

64 Hyodo F, Davis RM, Hyodo E, Matsumoto S, Krishna MC, Mitchell JB. The relationship between tissue oxygenation and redox status using magnetic resonance imaging. Int J Oncol 2012; 41: 2103-2108.

65 Vaupel P, Mayer A, Höckel M. Tumor hypoxia and malignant progression. Methods Enzymol 2004; 381: 335-354.

66 Sanchez-Alvarez R, Martinez-Outschoorn UE, Lamb R, Hulit J, Howell A, Gandara R et al. Mitochondrial dysfunction in breast cancer cells prevents tumor growth. Cell Cycle 2013; 12: 172-182.

67 Pérez-Escuredo J, Van Hée VF, Sboarina M, Falces J, Payen VL, Pellerin L et al. Monocarboxylate transporters in the brain and in cancer. Biochim Biophys Acta 2016; 1863: 2481-2497.

68 Martinez-outschoorn UE, Pavlides S, Howell A, Richard G, Tanowitz HB, Sotgia F et al. NIH Public Access 2012; 43: 1045-1051.

69 Whitaker-Menezes D, Martinez-Outschoorn UE, Lin Z, Ertel A, Flomenberg N, Witkiewicz AK et al. Evidence for a stromal-epithelial 'lactate shuttle' in human tumors: MCT4 is a marker of oxidative stress in cancer-associated fibroblasts. Cell Cycle 2011; 10: 1772-1783.

70 Fiaschi T, Giannoni E, Taddei ML, Cirri P, Marini A, Pintus G et al. Carbonic anhydrase IX from cancer-associated fibroblasts drives epithelial-mesenchymal transition in prostate carcinoma cells. Cell Cycle 2013; 12: 1791-1801.

71 Metallo CM, Gameiro PA, Bell EL, Mattaini KR, Yang J, Hiller K et al. Reductive glutamine metabolism by IDH1 mediates lipogenesis under hypoxia. Nature 2011; 481: 380-384.

72 Mullen AR, Wheaton WW, Jin ES, Chen P-H, Sullivan LB, Cheng T et al. Reductive carboxylation supports growth in tumour cells with defective mitochondria. Nature 2012; 481: 385-388.

73 Pérez-Escuredo J, Dadhich RK, Dhup S, Cacace A, Van Hée VF, De Saedeleer CJ et al. Lactate promotes glutamine uptake and metabolism in oxidative cancer cells. Cell Cycle 2016; 15: 72-83.

74 Krall AS, Christofk HR. Rethinking glutamine addiction. Nat Cell Biol 2015; 17: 1515-1517.

75 Corbet C, Draoui N, Polet F, Pinto A, Drozak X, Riant O et al. The SIRT1/HIF2a axis drives reductive glutamine metabolism under chronic acidosis and alters tumor response to therapy. Cancer Res 2014; 74: 5507-5519.

76 Boroughs LK, DeBerardinis RJ. Metabolic pathways promoting cancer cell survival and growth. Nat Cell Biol 2015; 17: 351-359.

77 Galiè M, Farace P, Nanni C, Spinelli A, Nicolato E, Boschi F et al. Epithelial and mesenchymal tumor compartments exhibit in vivo complementary patterns of vascular perfusion and glucose metabolism. Neoplasia 2007; 9: 900-908.

78 Afonso J, Santos L, Morais A, Amaro T, Longatto-Filho A, Baltazar F. Metabolic coupling in urothelial bladder cancer compartments and its correlation to tumor aggressiveness. Cell Cycle 2016; 15: 368-380.

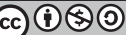

This work is licensed under a Creative Commons AttributionNonCommercial-ShareAlike 4.0 International License. The images or ther third party material in this article are included in the article's Creative Commons license, unless indicated otherwise in the credit line; if the material is not included under the Creative Commons license, users will need to obtain permission from the license holder to reproduce the material. To view a copy of this license, visit http:// creativecommons.org/licenses/by-nc-sa/4.0/

(c) The Author(s) 2017 